\title{
The Importance of Quality Assurance and Quality Improvement in Low- and Middle-Income Settings
}

\author{
Lipika Bhat ${ }^{1} \cdot$ Carol McCammon ${ }^{1}$ \\ Published online: 5 March 2021 \\ (C) The Author(s), under exclusive licence to Springer Science+Business Media, LLC part of Springer Nature 2021
}

\begin{abstract}
Purpose of Review This review will discuss the importance of quality improvement in low-and middle-income settings as well as several potential barriers to these measures.

Recent Findings There is substantial potential for growth in the field of quality improvement in low-resource settings. The extent of quality improvement (QI) research in low-resource settings is limited but expanding.

Summary Patient harm that is attributable to the costs of poor quality is a significant factor in all practice settings but is arguably of greater impact in limited-resource settings. Due to the recognized human, physical, and technological resource limitations in low- and middle-income countries, the delivery of quality healthcare can be variable in many settings, with discrepancies in evidence-based, evidence-informed, and customary care processes. Disparities in training and development of workforce providers exist in addition to limited availability of funding for QI research. Governmental, health ministry, and health system support is also variable. Attention to all of these areas is ultimately necessary to implement affordable and realistic quality improvement initiatives, education, training, and patient safety strategies that can mitigate harms, improve and establish more reliable outcomes, and develop a culture of safety to grow more sustainable and effective workforces and systems.
\end{abstract}

Keywords Quality · Low- and middle-income countries · Quality improvement $\cdot$ Patient safety $\cdot$ Culture $\cdot$ Sustainable

\section{Introduction}

There is a significant burden of harm that inherently results from medical care delivery which permits preventable adverse outcomes that injure patients and cause distress to their families and to the providers who delivered the care. Medical professionals around the globe are increasingly aware of this harm, and there is a growing knowledge base to systematically address underlying issues to create improved processes, systems, and protocols in continuous quality assurance (QA) and

This article is part of the Topical Collection on Global Health

Carol McCammon

mccammcf@evms.edu

Lipika Bhat

bhatl@evms.edu

1 Eastern Virginia Medical School, Emergency Physicians of Tidewater, 600 Gresham Drive, Raleigh Building, Rm 304, Norfolk, VA 23207, USA quality improvement (QI) efforts that enhance patient safety and favor superior outcomes. Fundamental phase transformation at all levels of healthcare delivery is needed to bridge the gap between good-quality care as defined by evidence-based and evidence-informed medicine, and what currently exists in practice. This complex continuum is constantly in evolution, which requires mindful focus, stepwise process changes, and endless dedication to a culture of patient safety and quality care that adds value and attains realization of improvement. Additionally, reliable systems of quality healthcare incentivize capable and qualified individuals to stay in their home communities.

In 1999, the Institute of Medicine published the landmark report To Err is Human that disclosed how tens of thousands of patients die each year in the USA as a result of medical error [1]. This report caused a monumental shockwave of concern, not only in the public sector, but also in the epicenter of US medicine to begin to systematically examine the current medical practice to design reforms to mitigate this disturbing reality. It was recognized in the book Crossing the quality chasm: a new health system for the 21st century (2001) that reform at 
the margins of the US healthcare system as it existed could never be effective enough to change the preventable harms of medical error and poor quality. A comprehensive vision of the future guided by the six aims of medical quality was necessary to fundamentally address the pervasive problems of the entire system. These six aims as established at that time are that medical care should be safe, effective, efficient, patient-centered, timely, and equitable [2]. Embedding a culture of quality improvement and patient safety within all health systems around the world can assist in work towards these aims to mitigate harm. There are multiple levels of interventions that can improve quality of care and patient safety. It is important to remember that sweeping changes across systems, municipalities, nations, and global landscapes take time and can be well-supported by close attention to patient safety that is within reach at the local level in the present moment.

\section{Importance of Quality in LMIC}

While interventions in low- and middle-income countries are often focused on expanding access to care, including specialized technology and medicine, it is vital to consider not only the quantity of care delivered but also the quality. Even where there is a broad access to healthcare services, if those services are perceived to be of low quality, demand and therefore healthcare coverage will remain low [3]. As noted by Leatherman et al., "the gap between the actual care delivered and the best possible care is often much greater" in lowresource compared to high-resource settings, so narrowing that gap via QI can have an even greater impact in low-and middle-income countries (LMICs) [4••].

High-quality care reflects appropriate stewardship of resources. When resources are limited, it is all the more important that they are utilized in the safest, most efficient, and most equitable manner possible. Quality improvement can minimize waste of resources and maximize equitable outcomes, including achievement of Millennium Development Goals [4.•], which promotes confidence in the health system and encourages further government and donor investment into the system. Additionally, QA practices can improve the consistency of care throughout a given system, by elimination of poor-performing outliers and raising the standard overall.

It is crucial that QI also be recognized as important by the local population and that it is not based solely on the values of an NGO donor, or performed as a checkbox requirement for funding [5]. There is a persuasive need for a shared vision of QI with the goal of improving care and ensuring patient safety to the best degree possible.

Additionally, reliable methods to gather data on quality measures as well as on population demographics are contributory to the process. Quality cannot be improved if it cannot be identified [5]. While there may be barriers to the collection and dissemination of QI efforts and data due to resource constraints, there exists "an obligation to assure that quality improvement science is relevant not just in high-income countries, but wherever improvement might reduce suffering and mortality" [6].

\section{QI Defined}

An assessment of the current state of the quality of care delivery is essential to understand in order to begin to design potential improvement efforts to attain the desired quality of care. First, a solid and useful definition of healthcare quality is paramount to QI efforts. Kashmer et al. provide this practical definition: "Healthcare quality improvement is the systematic reduction of the variation in output of a system, sometimes accompanied by adjusting the central tendency of the system, as measured against specification limits from the third-party payer, patient or other important recipient of the system output - whichever is the most difficult to satisfy" [7]. This definition emphasizes a standard approach to QI that targets consistent acceptable results. There are reliable QI tools already in existence that are useful in service and manufacturing industries. They are applicable in all functions of QI, no matter the industry or product, and application of these tools in healthcare is well-established. It is also essential to understand that every output from a system creates a distribution which may or may not be a mathematically normal distribution. In thinking of quality in this way, it is desirable to have comparable and acceptable outcomes for each output of the care process. It is essential to recognize that a change in central tendency alone is not always an indication that quality has improved; simply looking at an average may not solve the problem because the average itself does not represent consistency or acceptability in outcomes generated. Consistency and reliability of acceptable outcomes is key, not simply the system average. Most local-level quality efforts focus on the patient as the most important and difficult to satisfy recipient and stakeholder in delivery of healthcare. Other stakeholders must be considered as well, including the caregivers; local, regional, and national systems; government and nongovernmental organizations, and the communities served.

\section{General Approach to QI}

A systematic approach to QI using established QI methodology and tools can assist organizations and departments to identify attainable changes that achieve desired outcomes and close the gap between the Current State of wide variability in system output and the Desired Outcome of reliable and consistent acceptable results. 
For a very simple theoretical example, in the treatment of fever, it is desirable to have a reduction in the patient's temperature as the outcome. Say there are multiple treatments being employed with varying results, with some doctors that prescribe acetaminophen, some prescribe tepid water bath, and some prescribe bundling in blankets. It was noted that a wide distribution of outcomes existed in the current state, and the question was posed whether this could be improved for better fever control for the patients. The interested team evaluated the three treatments and their outcomes retrospectively for the past 3 months through a chart review. They found that the Current State of practice was adequate fever reduction in $95 \%$ of the patients who received acetaminophen, $50 \%$ of patients who received tepid water bath, and $0 \%$ of patients who were bundled in blankets. The team determined that the Desired Outcome was to attain adequate fever control in $>$ $90 \%$ of febrile patients in 3 months, defined as reduction in temperature to $<38{ }^{\circ} \mathrm{C}\left(<100.5^{\circ} \mathrm{F}\right.$.) A well-established QI tool, Deming's Cycle, was utilized to plan their QI project.

Deming's Cycle, also referred to as PDSA Cycle (Plan, Do, Study, Act) was used to conceive a QI project to improve fever outcomes, with a goal aimed to adequately reduce fever in $>90 \%$ of febrile patients in 3 months. The team carefully started the Plan portion of a QI project based on the retrospective Current State data. The intervention involved education of the ordering providers to use properly dosed acetaminophen in appropriate patients as first-line fever reduction and eliminate ineffective bundling in blankets as an option. They would track the same outcomes for 3 months once this education was disseminated. The team then did the Do portion, distributed the education, and subsequently began to track the outcomes prospectively. As the data was being collected, the team noted more consistent results with adequate fever reduction in $80 \%$ of patients by the end of the third month. They were concerned that they did not attain their set goal of $>90 \%$, but moved ahead to the Study part of the cycle, in which the team recognized that in the $20 \%$ of patients with inadequate fever reduction, tepid water baths alone were prescribed. This led the team to Act on these results and adjust the project to recommend acetaminophen be utilized as the first-line monotherapy in all appropriate patients with fever, and a tepid water bath could no longer be used as a single intervention unless there was a contraindication to acetaminophen. The team decided to track the outcomes for 6 weeks this time, and at the end of that time period, they found that $94 \%$ of treated patients had attained adequate fever control. The team shared this very positive feedback with all who worked to change their practice and contributed to the success of the project. This was a sustainable change as the providers bought into the project and effectively altered their practice in the long term with improved patient fever control outcomes. The most important message from this example is that this PDSA tool is applicable and adjustable to any clinical improvement initiative.

\section{Indicators and Implementation of Quality in LMIC}

Indicators and/or target measures for quality may differ in low- and middle-income compared to higher income countries due to the patterns of disease and resource access. Low- and lower-middle-income countries still have a much higher rate of maternal and pediatric (under 5) mortality compared to upper-middle-income countries, as well as a greater proportion of deaths from communicable diseases or nutritional conditions, as compared to higher income countries with prevalent noncommunicable diseases [8••]. These considerations are paramount not only to medical outcome improvement, but also to surgical outcome improvement through maximization of prenatal and perinatal care, and optimization of nutritional conditions when possible for all patients preoperatively for optimal results.

While quality improvement has been identified as a key component of better health outcomes, it must be acknowledged that systems and programs that work in one setting may not be directly transferable or generalizable to another. Evidence-informed care based on current science that supports the delivery setting and local resources may be the ultimate goal, with a vision for continuous QI efforts and dedication to patient safety. Successful approaches are often multimodal, addressing providers, patients, and the systems within which they encounter each other simultaneously [4••].

Ruelas et al. proposed a series of questions as part of a framework that may be used to assess local contextual, strategic, and structural factors that could affect QI implementation in low- and middle-income countries. These include consideration of the values, needs, and expectations of the population, government and healthcare leaders, and donors, and how these can be aligned; the availability of funding; knowledge of basic QI concepts and operational definitions; capacity of the health system to meet population needs; availability of adequate information systems for measurement and reporting; and sustainability of proposed change $[8 \cdot \bullet]$.

\section{Challenges for QI Initiatives in LMIC}

Measurement of technical quality of care is decidedly important, but additional factors also need consideration in planning and implementing QI. These considerations include examination of the process of care at multiple points through the care pathway, patients' perceptions of quality as compared to the actual clinical quality, social norms, accessibility, trust, and acceptability of care offered in comparison to other options. The World Health Organization identified six areas of challenge in the concept and measurement of quality of care in low- and middle-income settings. Even though patient perception is not a direct or reliable measure of quality, their 
perceptions significantly influence their utilization of accessible care. The individual's experience occurs over a period of time and is relevant in all care encounters, so quality must be pursued consistently in all episodes of care. Thirdly, responsiveness of the providers and the system to the patient's needs is significant. Management at the facility, regional, state, and national levels deeply affects quality of care and how it is regarded. Different aspects of the community come together to produce the social construct of quality in healthcare services and should be considered in QI efforts as appropriate to the goal of each project. Finally, approaches to quality measurement in low- and middle-resource communities may require adaptation for utility in the setting and eventual enhancement to more readily support broader QI efforts as experience develops [3].

Limited economic settings are notably resource-challenged in both physical assets such as hospital beds, personal protective equipment, technology, and human capital. However, even with a great amount of variation between countries and regions of countries within the same income classification, there are opportunities to harness. Within such constraints, innovative solutions are required for enhancing quality tailored to local circumstances.

Many projects require Institutional Review Board or Ethics Committee review prior to starting a quality project. Although specific protected patient information is not the usual focus of quality improvement projects, there are times when approval may be delayed based on the protection of health information. Movement to mitigate full reviews and utilization of a "not human subjects" investigation category can be helpful, as most quality improvement is evaluated on a systems or local departmental basis, rather than on individuals. This can be conducted without potential for compromise of protected personal health information or harm resulting from traditional research methodology.

Quality improvement projects that are undertaken are frequently not published, and there exist "few reliable routes of site-to-site or nation-to-nation shared learning" [4・•]. Publications related to quality in LMIC also tend to be descriptive instead of hypothesis-driven, which may limit applicability to other settings and potential for building upon QI science [6]. As QI knowledge, experience, and interest are not homogeneously spread, collaboration is imperative. Expanding the body of research in this field and facilitating knowledge exchange could allow for more rapid development and implementation of QI methods globally.

In lower income countries, robust evidence-based national guidelines that may be used as a standard to evaluate clinical practices may not exist, so the quality measures captured in this way might be limited [3]. Data collection and reporting processes may also be limited in LMICs and require significant modifications to ensure completeness and accuracy, so implementation of any QI project may be delayed $[5,9 \bullet \bullet]$.
Additionally, initiatives may be hindered by poor conceptualization of the technical aspects of the QI process as well as by competition with other global health programs $[4 \bullet \bullet]$. There may be a tight balance required between highlighting QI as a particularly important process that requires careful attention, which could prevent it from becoming simply another program among many, as contrasted to cultivation as an essential pillar of the healthcare delivery system.

Lack of funding is frequently cited as a barrier to QI projects $[4 \cdot \bullet, 9 \bullet \bullet]$. Further, recruitment of the manpower to administer quality improvement may seem challenging and difficult to champion. However, when viewed as a mechanism to evaluate the effectiveness of care processes, even slight changes can result in big improvements that save cost in resource utilization which ultimately improves care, simplifies the physicians' responsibilities, decreases iatrogenic injury, improves diagnostic accuracy, supports evidence-informed treatment appropriate in the setting, decreases surgical site infections, and decreases complications requiring hospitalization or rehospitalization among many other foci. This adds to the value of making quality improvement a priority, no matter the setting.

Strong leadership and governance are necessary to ensure the success of a QI plan, as with any health initiative. Political instability at the national level, or leadership turnover at a regional or even institutional level, can have detrimental effects on healthcare quality $[9 \bullet \bullet]$. Management practices that incorporate methods to reward quality and correct or sanction poor performance can strengthen QI initiatives [10].

Finally, perceptions are important. Patients will base health-seeking behavior on their perception of quality, which may not correlate with the actual quality of care provided. This may be related to perceived or tangible discrimination, past experiences, or preconceived notions about superiority or inferiority of care delivered in a particular location (e.g., rural vs. urban, public vs. private) [3]. The ultimate aim of QA and QI is to benefit the patient, so a foundation of trust is key to matching perceptions with reality.

\section{Looking to the Future}

Health systems, physicians, and staff are faced with real-time challenges that demand appropriate and safe care for each patient while ethically balancing the use of resources at hand to be sure that comparable care is available for others in need, while protecting the integrity of the caregivers' well-being.

In recent times, this lesson has manifested in paramount proportions from the SARS, MERS, and Ebola outbreaks, and currently in the COVID-19 pandemic. Application of resources at hand demands good stewardship that bases the care of each patient on their medical needs, makes decisions with accurate scientific evidence in mind when available, and 
chooses actions that utilize the fewest resources with the likelihood of the safest outcomes for most [4••]. It is certainly an illuminating observation of COVID-19 that the highest resource nations are struggling with equitable distribution and utilization of resources. Those who have learned these lessons well have great contributions to offer. There is much to be gained from bidirectional collaboration that supports unity in the aims of healthcare quality in all resource settings. No resources are infinite.

Quality improvement efforts in limited-resource settings do not need to be complex or require significant financial input or systems overhaul. Sometimes a small change can lead to a large adjustment in creating a culture of safety. For example, a simple surgical checklist is a low-cost and effective intervention that has shown demonstrable decreases in clinical errors and mortality. As Internet and mobile phone connectivity continues to grow globally, the development of opensource electronic medical record programs and patient outreach via web-based phone applications or SMS messaging provide opportunities to harness technology to facilitate data collection, communication, and patient engagement [11].

QI research efforts in limited-resource settings may also be supported by enhancing mentorship programs for trainees, creating options for sharing QI efforts via outlets other than peer-reviewed journals, and nurturing a network of researchers to enable the sharing of experiences and collaboration to develop solutions [6]. Multiple open-access free platforms are available to enhance collaboration.

\section{Conclusion}

Provision of safe, effective, and efficient care that is timely, equitable, and patient-centered is a reasonable aim that physicians ascribe to, which mirrors the reasons why most enter the profession. Physicians, as the experts, must own patient safety and quality, create the basis for incorporating quality assurance and improvement into existing systems, and embed this knowledge and aspiration into the training of rising professionals. Physicians, as the leaders, are responsible for cultivation of work environment values and ethos; thus, a perspective that encompasses QI as a valued competency of medical practice can unify all in the advancement of a focused culture of patient safety and continuous improvement. In low-resource settings, even small changes can have an outsize impact, and the field of quality and safety presents increasing opportunities for essential research and growth.

\section{Compliance with Ethical Standards}

Conflict of Interest The authors declare that they have no conflict of interest.
Human and Animal Rights and Informed Consent This article does not contain any studies with human or animal subjects performed by any of the authors.

\section{References}

Papers of particular interest, published recently, have been highlighted as:

•• Of major importance

1. Institute of Medicine. In: Kohn L, Corrigan J, Donaldson M, editors. To err is human. Building a safer health system. Washington, DC: The National Academies Press; 1999. https://doi.org/10. $17226 / 9728$.

2. Institute of Medicine. Crossing the quality chasm: a new health system for the 21 st century. Washington, DC: National Academies Press; 2001. https://doi.org/10.17226/10027.

3. Hanefeld J, Powell-Jackson T, Balabanova D. Understanding and measuring quality of care: dealing with complexity. Bull World Health Organ. 2017;95:368-74. https://doi.org/10.2471/BLT.16.179309.

4••. Leatherman S, Ferris TG, Berwick D, Omaswa F, Crisp N. The role of quality improvement in strengthening health systems in developing countries. Int J Qual Health Care. 2010;22(4):237-43. https:// doi.org/10.1093/intqhe/mzq028 Reviewed proceedings from 2008 Bellagio, Italy QI conference, highlighting barriers to and recommendations for strengthening QI initiatives globally.

5. Bradley EH, Yuan CT. Quality of care in low- and middle-income settings: what next? Int J Qual Health Care. 2012;24(6):547-9. https://doi.org/10.1093/intqhe/mzs065.

6. Garcia-Elorrio E, Schneider EC. Research on health-care quality improvement in low- and middle-income countries: is it a worthy investment? Int J Qual Health Care. 2012;24(6):550-2. https://doi. org/10.1093/intqhe/mzs067.

7. Kashmer D, Cannon S, Wolf M, Gray J, Hayne W. Ten secrets: a healthcare quality improvement handbook: The Healthcare Lab, Inc; 2018.

8••. Ruelas E, Gómez-Dantés O, Leatherman S, Fortune T, Gay-Molina JG. Strengthening the quality agenda in health care in low- and middle-income countries: questions to consider. Int J Qual Health Care. 2012;24(6):553-7. https://doi.org/10.1093/intqhc/mzs061 Proposed strategic questions to assess and strengthen QI agenda in LMIC.

9••. Magge H, Kiflie A, Nimako K, et al. The Ethiopia healthcare quality initiative: design and initial lessons learned. Int J Qual Health Care. 2019;31(10):G180-6. https://doi.org/10.1093/intqhe/mzz127 The Ethiopia Health Care Quality Initiative (EHCQI) demonstrated initial successes and identified challenges in implementing the prototype phase of a national health system improvement project.

10. Topp SM, Chipukuma JM, Hanefeld J. Understanding the dynamic interactions driving Zambian health centre performance: a casebased health systems analysis. Health Policy Plan. 2015;30(4): 485-99. https://doi.org/10.1093/HEAPOL.

11. Eyob B, Boeck MA, FaSiOen P, Cawich S, Kluger MD. Ensuring safe surgical care across resource settings via surgical outcomes data and quality improvement initiatives. Int J Surg. 2019;72:2732. https://doi.org/10.1016/j.ijsu.2019.07.036.

Publisher's Note Springer Nature remains neutral with regard to jurisdictional claims in published maps and institutional affiliations. 